\title{
Aspects on the Dynamics and Detection of Additional Circumbinary Extrasolar Planets
}

\author{
Tobias C. Hinse ${ }^{1,2}$, Nader Haghighipour ${ }^{3}$ and Krzysztof Goździewski ${ }^{4}$ \\ ${ }^{1}$ Korea Astronomy \& Space Science Institute, 305-348 Daejeon, Republic of Korea \\ ${ }^{2}$ Armagh Observatory, College Hill, BT61 9DG, Armagh, UK \\ email: tchinse@gmail.com \\ ${ }^{3}$ Institute for Astronomy \& NASA Astrobiology Institute, University of Hawaii, 96822 HI, USA \\ ${ }^{4}$ Torun Center for Astronomy, Nicolai Copernicus University, Torun, Poland
}

\begin{abstract}
The presence of additional bodies orbiting a binary star system can be detected by monitoring the binary's eclipse timing. These so-called circumbinary objects will reveal themselves by i) either introducing a reflex motion of the binary system about the total system's barycenter creating a geometric light-travel time effect (LITE), ii) by gravitational perturbations on the binary orbit, or iii) a combination of the two effects resulting in eclipse timing (ETV) and transit timing (TTV) variations. Motivated by the four recently detected circumbinary planets by the Kepler space telescope (Kepler-16b, Kepler-34b, Kepler-35b and Kepler-38b) we have begun to study their dynamics in the presence of an additional massive perturber. In particular we used Kepler-16b as a test bed case. We are aiming to study the detectability of non-transiting and inclined circumbinary planets using the ETV effect along with the fast indicator MEGNO to quantitatively map the chaotic/quasi-periodic regions of the orbital parameter-space and to determine where the orbit of a circumbinary planet will be stable. We have calculated the amplitudes of TTV and ETV signals for different values of the mass and orbital elements of the planet and binary.
\end{abstract}

Keywords. binaries: close, eclipsing, subdwarfs - methods: data analysis, numerical - planets and satellites: detection, dynamical evolution and stability

\section{Introduction}

Recently, using the Kepler space telescope, several single planet (and multi-planet system Kepler-47b,c, see the paper by Welsh et al. in this proceedings) systems (Kepler16b, Kepler-34b, Kepler-35b \& Kepler-38b) have been detected orbiting a binary star system (Doyle et al. 2011, Welsh et al. 2012, Orosz et al. 2012a). These planets have been detected by the transit technique in which the planet (or planets) causes a decrease in the observed brightness of one of the components in the binary. However, other techniques exist to detect additional massive objects orbiting a binary star system. Up to this time, the most successful technique that relies on the finite speed of light. In the case of a single isolated binary system the distance between the binary masscenter and the observer remains constant. However, if an additional body orbits the binary system the binary mass centre will describe an orbit around the system barycenter giving rise to the so-called light-travel time effect (LITE) (Irwin 1952). This effect is a special case of the more general eclipse timing variation effect and is a pure geometric effect. Period changes or eclipse timing variations can also be caused by direct gravitational perturbations of an additional massive object on the binary orbit or due to interactions between the binary components (magnetic interactions or mass transfer).

The variation in distance of the binary relative to the observer translates into a periodic change in the measured eclipse times of the primary and secondary eclipses. The binaries 
ephemeris will then be described by a quasi-periodic variation in addition to the linear part. Removing the linear part obtained from a least-squares fit to the observed eclipse times results in the so-called $O-C$ diagram. Any variation in the $O-C$ may provide clues about period changes in the binary orbital period and its physical nature/cause. The period changes could be due to the interaction between the two binary components giving rise to a secular increase or decrease of the binary orbital period. However the eclipse period can also be modulated by additional circumbinary companions giving rise to quasi-periodic variations.

Recently, single and multi-planet circumbinary companions has been proposed from ground-based observations for some years. Lee et al. (2009) were the first to interpret the $O-C$ variation of the short-period eclipsing binary HW Virginis (HW Vir) as a multiplanet system. Their discovery were subsequently followed by several other detections of cirumbinary companions from the detected LITE signal (Schwarz et al. 2009, Beuermann et al. 2010, Potter et al. 2011, Qian et al. 2011) and very recently (Almeida et al. 2013). However, for some systems the initially proposed models produced orbital architectures and companion masses to be highly unstable (Horner et al. 2011, Hinse et al. 2012, Horner et al. 2012a,b, Beuermann et al. 2012, Wittenmyer et al. 2012, Wittenmyer et al. 2013). In their recent analysis Goździewski et al. (2012) established with some confidence the existence of the inner companion of the multi-planet system hosted by HU Aquarii (HU Aqr) binary. In addition, Beuermann et al. (2013) showed stable orbits for the companions orbiting the NN Serpentis binary system.
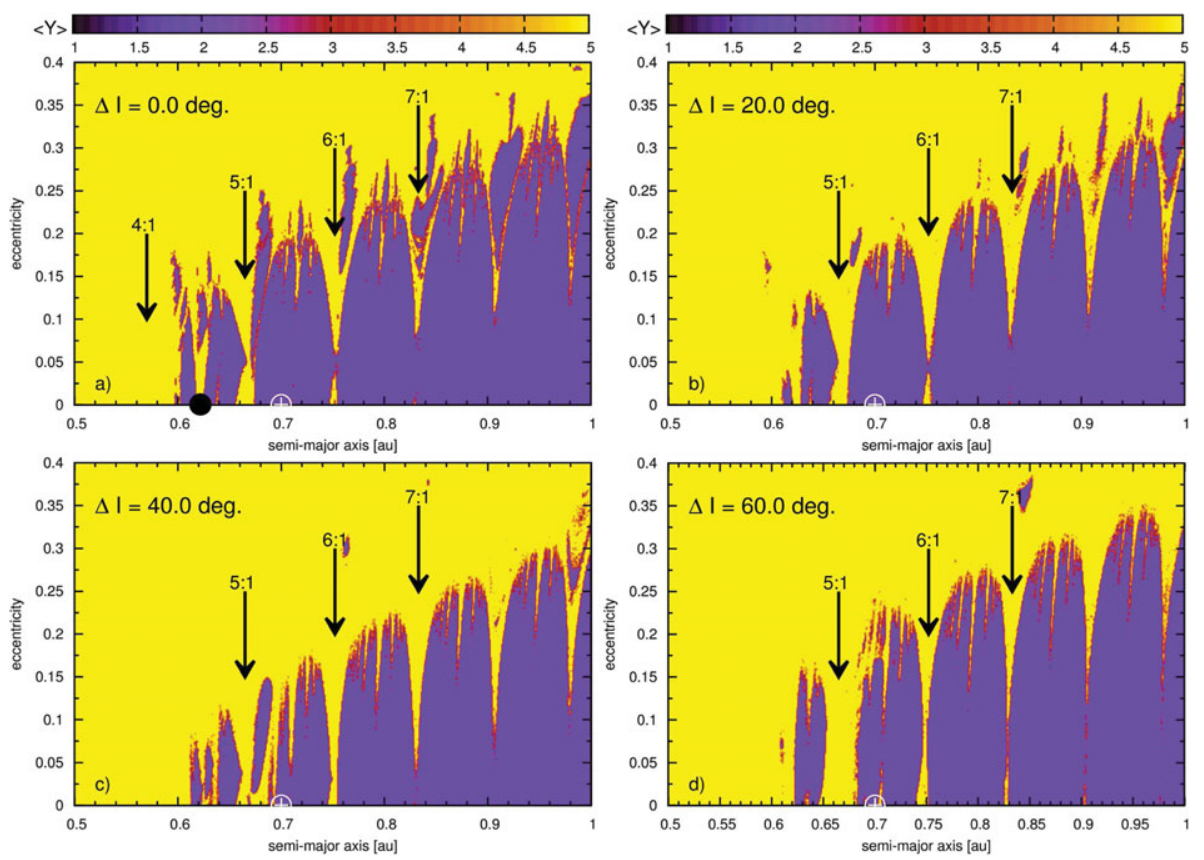

Figure 1. Dynamical maps of the Kepler-16b system with MEGNO color coded in the interval $1\langle\langle Y\rangle\langle 5$. Quasi-periodic orbits have $\langle Y\rangle \simeq 2.0$ and appear blue (or dark). Chaotic orbits appear in as yellow (light) colors. We show the results of four different orbital inclinations of Kepler-16b relative to $I=90^{\circ}$. The planet (Kepler-16b) has orbital parameters $(a, e) \simeq(0.7 \mathrm{au}, 0.0)$ and its location is marked by a white cross. The locations of mean-motion resonances are shown by arrows placing the planet between the 5:1 and 6:1 resonance. In panel-a the black circle marks the location of an initial condition close to the 9:2 resonance. The resolution of the map is $\left(N_{x}, N_{y}\right)=(500,300)$ pixels. See electronic version for color figures. 

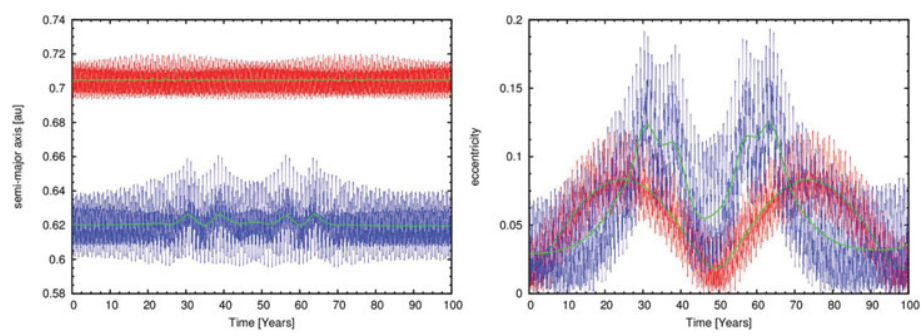

Figure 2. Time evolution of orbital elements for a quasi-periodic and chaotic orbit. Initial conditions are shown in Fig. 1a. Left panel: semi-major axis vs time. Right panel: eccentricity vs time. The eccentricity for the quasi-periodic exhibits a secular period of about 50 years. The chaotic orbit shows a clear excitation in eccentricity characteristic for motion in MMR. See electronic version for color figures.

This paper is structured as follows. In section 2 we consider the three-body problem and study the dynamics of a single circumbinary planet on an inclined orbit. We use Kepler-16 as a test case and study the qualitative change in the topology of the $(a, e)$ phase space for different orbital inclinations. We will apply an efficient chaos indicator (measuring the maximum Lyapunov exponent) to study the time evolution of a few tenth of thousand initial conditions around the measured orbital parameters of Kepler-16b. For inclination where the planet is no longer transiting the binary stars, we calcualted the corresponding LITE signal. In section 3 we then add another planet to the Kepler- 16 system and study the dynamics due to the additional perturbations. The resulting LITE, ETVs and TTVs effects are calculated and studied and compared to each other in section 4 . In section 5 we provide conclusions.

\section{Dynamics of Kepler-16b on inclined orbits}

The Kepler-16b system (Doyle et al. 2011) is essentially a three-body system possibly exhibiting complex dynamical behaviour. The first question to address is the planet's orbital stability around the binary. We have therefore used the MEGNO† (Cincotta \& Simó 2000, Cincotta, Giordano \& Simó 2003, Gozdźiewski et al. 2001, Slonina et al. 2012) tool to detect chaotic regions. Especially we consider the semi-major axis and eccentricity space $(a, e)$ of the planet. We implemented the full $N$-body equations of motion and explore various orbits of the involved bodies in the Jacobian reference frame. Therefore, the planets semi-major axis is relative to the barycenter of the binary pair. The binary orbit is fully resolved during the numerical integrations. Typical integration times span around $12 \times 10^{3}$ binary periods.

In Fig. 1a, we show the result for the nominal solution as presented in (Doyle et al. 2011) with Kepler-16b on a near co-planar orbit with the binary plane. We color code yellow (or bright) regions as chaotic dynamics and blue denoting quasi-periodic (regular) orbits. The semi-major axis of Kepler-16b is around 0.7 au and places the planet well separated from the general instability region surrounding the sligly eccentric binary orbit. Identifying the locations of mean-motion resonances (MMRs) between the binary and planetary orbits, we also note that Kepler-16b is located between the 5:1 and 6:1 MMR. The dynamics of the planets long-term time evolution might be governed by the 11:2 MMR. As an example, we have highlighted the difference between the time evolution of a quasi-periodic and a chaotic initial condition (Fig. 1a). We mark the location of an initial condition close to the 9:2 MMRs in the $(a, e)$ phase space (see black circle in Fig. 1a). 

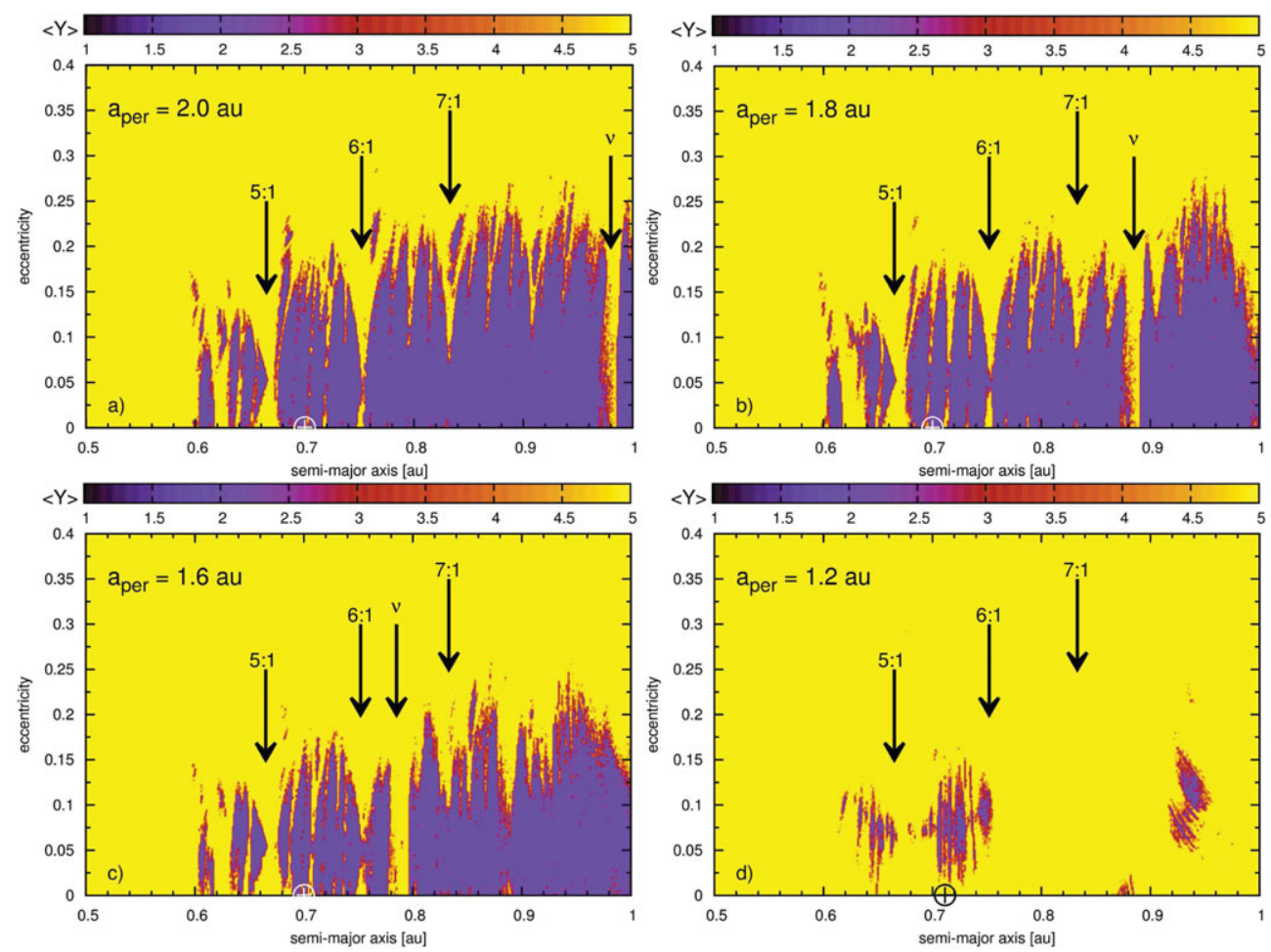

Figure 3. Dynamical maps of the Kepler-16b system perturbed by an outside perturber of mass $5 M_{j u p}$. Color coding as in Fig. 1. We show the results of four different orbital semi-major axis of the perturbing orbit. Kepler-16b has orbital parameters $(a, e) \simeq(0.7 \mathrm{au}, 0.0)$. The locations of mean-motion resonances are shown by arrows placing the planet between the $5: 1$ and $6: 1$ resonance. The location of the secular resonance $(\nu)$ sweeps towards Kepler-16b as the orbit of the pertuber is decreased. In the lower right panel almost all phase space is dominated by chaotic dynamics. See electronic version for color figures.

In Fig. 2 we show and compare the time evolution of the planets eccentricity and semimajor axis for two different initial conditions. Chaotic time evolution is readily visible in the excitation of the eccentricity for the initial condition starting close to the 9:2 MMR. This evolution is to be contrasted with the quasi-periodic orbit corresponding to the nominal solution of Kepler-16b showing a periodic change in the eccentricity with a secular period of around $\sim 50$ years. We have also considered the case of various inclinations of the planets orbit relative to the binary orbital plane. Placing the planet on various inclined orbits we calculated MEGNO maps for each case and show our results in Fig. 1b to Fig. 1d. For highly inclined orbits the inner instability region extends to larger semi-major axis of a circumbinary companion. This extension of instability is most likely introduced by the Kozai mechanism (Kozai 1962) decreasing the planets periastron as a result of coupling the orbital inclination and eccentricity with large amplitude oscillations in anti-phase. Surprisingly, our results suggest, that the orbit of Kepler-16b would exhibit a quasi-periodic character and remain stable for an inclined orbit as high as $60^{\circ}$.

\section{Two-planet circumbinary dynamics}

Detecting additional companions orbiting a binary is possible either via the transit method (see the paper by Welsh et al. in this proceedings and the paper by Orosz et al. $2012 \mathrm{~b}$ ) or by recording the eclipse timing variations over a sufficiently long observing 

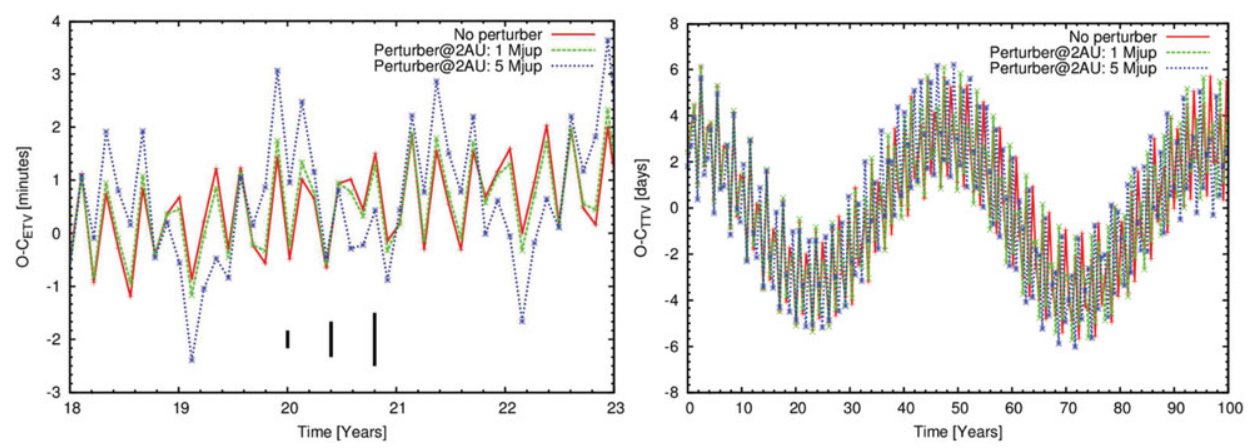

Figure 4. Results of calculating the $O-C$ diagrams for different masses of the outside perturber. Left panel: Eclipse-timing variations of the binary orbit. Right panel: Transit timing variations of the inner planet. The electronic version for color figures.

baseline. The latter technique has the advantage to detect non-transiting circumbinary companions; though only the minimum mass of the companion and minimum semi-major axis of the corresponding LITE orbit can be inferred from timing measurements. We have simulated a two-planet system by adding an additional planet to the Kepler-16b system. We considered various masses of the extra hypothetical planet and started the pertuber on an outer $(a>1.2 \mathrm{au})$ near-circular orbit. Results of calculating the MEGNO factor for the four-body problem are shown in Fig. 3 for various initial semi-major axes of the outside perturber starting from $a=2.0$ au to $a=1.2 \mathrm{au}$. The dynamical topology of the $(a, e)$-phase space now changes dramatically compared to the single-planet case. Upon close inspection of Fig. 3a - Fig. 3d the most prominently change is the introduction of the secular resonance $\nu=\omega_{1}-\omega_{2}$ (Murray \& Dermott 2000) between the two planets apsidal frequencies. It is also found that considering a $5 M_{J u p}$ planet on a $a=2.0$ au orbit would not dramatically endanger the inner Kepler-16b planet. Most of the phase space neighbourhood around Kepler-16b remains to exhibit quasi-periodic orbits. However, as the outer perturber is moved inwards, the location of the secular resonance sweeps across the interval $[0.9,1.1]$ au. For single star planetary systems, additional planets on outer orbits are known to have significant effects on the dynamics of a possible planet within the habitable zone (Migaszewski et al. 2008) due to the $\nu$ resonance.

\section{Detecting an additional planet via timing measurements}

We also implemented the calculation of ETVs and TTVs during the numerical integration due to the additional perturber. In the presence of an outside massive perturber the inner transiting planet will start to exhibit TTVs as its orbit experiences perturbations (Agol et al. 2005). In addition, the binary will have an additional sinusoidal component in the $O-C$ eclipse timing variation due to the light-travel time effect. A single circumbinary companion will cause a sinusoidal-like variation superimposed on the linear ephemeris of the binary period. In Fig. 4 we show the result of calculating the ETV and TTVs signals for three different masses of the perturber $\left(0,1,5 M_{j u p}\right)$. The semiamplitude in the ETVs (left panel of Fig. 4) increases with increasing mass with a ETV semi-amplitude of around $\simeq 3$ minutes. Remarkably, the TTV semi-amplitude (left panel in Fig. 4) can be as large as 6 days when the system is observed over a total time period of about 50 years. However, additional planets are most likely detected from the induced ETV signals. Close-in, massive companions are most likely to be detected first during a dedicated follow-up observing campaign.

We have also calculated the resulting ETV due to the light-travel time effect from the numerical integration. We calculated the $z$-component of the binary barycenter relative to 

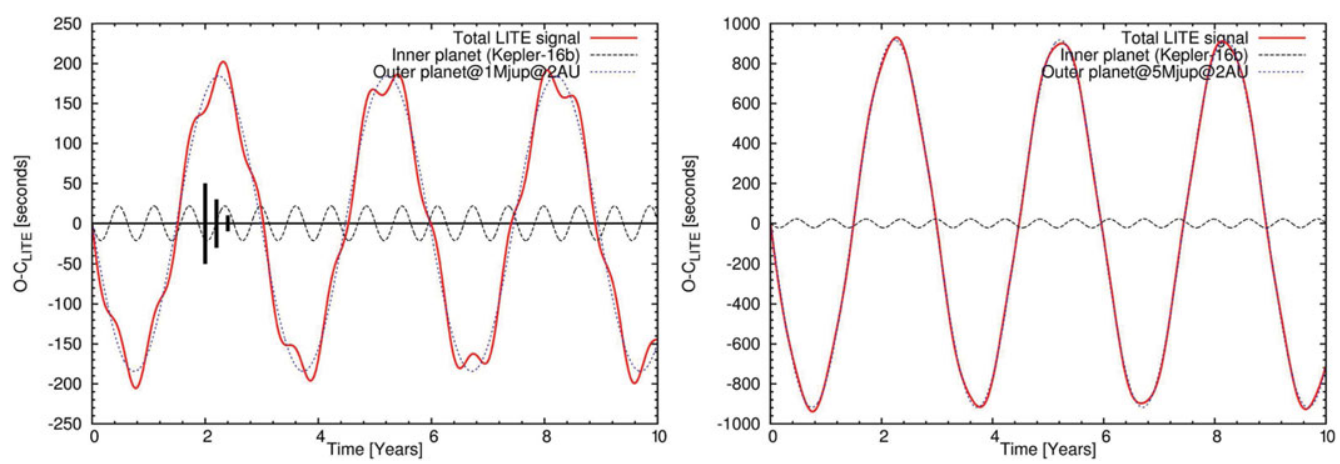

Figure 5. The result of calculating the LITE effect $(-z / c)$ for a single planet like Kepler-16b in combination with an additional outer pertuber considering two different masses ( 1 and $5 M_{j u p}$ ). Left panel: The small amplitude variation is due to Kepler-16b only. The large amplitude variation is due to the outer perturber of $1 M_{\text {jup }}$. The solid curve is the total signal. Vertical bars show 1- $\sigma$ errorbars of $\pm 10,30$ and 60 seconds. Right panel: The total LITE signal due to an additional planet with mass $5 M_{j u p}$, but same semi-major axis. The electronic version for color figures.

the total system barycenter from the positions and masses of the companions. The timing variations are then calculated from $-(z / c)$ where $c$ is the speed of light. We calculated the LITE signal from Kepler-16b as a stand-alone system and also for two masses (1 and $5 M_{\text {jup }}$ ) of an outer perturber on a $a=2.0$ au orbit. We present our results in Fig. 5. The left panel exhibits a small-amplitude ( $\simeq 25$ seconds) LITE variation caused by Kepler-16b and a larger amplitude variation caused by the additional object of mass $1 M_{j u p}$. The overall combined LITE signal is shown as a solid line with a semi-amplitude dominated by the perturbing body. In the right panel of Fig. 5 we have increased the mass of the perturber by a factor of 5 . We have the following remarks. First, in order to characterise or constrain the additional object from the observed LITE signal one would need timing precision of around 10 seconds (see Fig. 5 caption for details) in order to catch the smaller "wiggles" as introduced by Kepler-16b. We note the danger of missing additional inner companions. The mass and orbit of the additional perturber will be well characterised from the LITE amplitude and period as the $O-C$ diagram is populated with timing measurements. However, the right panel demonstrates that for increasing mass of the outer companion the inner companion might be missed due the much larger amplitude of the much more massive perturber. It is possible that the inner companion (Kepler-16b) can still be detected via TTVs of the outer perturber, if such transit signals are observed. We plan to investigate this in more detail in the future.

\section{Conclusions}

Using MEGNO we have investigated the dynamics of Kepler-16b on various inclined orbits. We found that even for highly inclined orbits, the planet would exhibit a quasiperiodic variation in phase space suggesting a stable orbit. Though the planet is then no longer transiting, such a system might be detectable from ETVs of the binary period. We then investigated the dynamical changes when an outside perturber is introduced to the Kepler-16b system. We calcualted dynamical MEGNO maps for various masses and distances of the perturber and detected the presence of a secular resonance between the two planets. As the orbit of the outside pertuber is decreased the location of the secular resonance decreases as well. This might have dynamical consequences for an additional planet in the planetary system of Kepler 16. Considering an additional massive perturber 
we then investigated its detectability via ETV, TTV and LITE. We found that additional planets on wider orbits are most likely to be detected via their ETVs. However, if the mass of the pertuber is too high we found that additional inner planets might be undetected as their LITE signals will be diminuished.

\section{Acknowledgements}

We thank the referee peer-reviewing and careful reading of the paper providing constructive and helpful comments which significantly improved the initial presentation. TCH acknowledges financial support from the Korea Research Council for Science and Technology (KRCF) in form of the KRCF Young Scientist Research Fellowship and financial support from Korea Astronomy and Space Science Institute (KASI) grant number 2012-1-410-02. In addition, TCH acknowledges a generous financial support by the IAU (grant ID: GA922). Numerical simulations were performed at the SFI/HEA Irish Centre for High-End Computing (ICHEC) and the PLUTO computing cluster at KASI. KG is supported by the Polish Ministry of Science and Higher Education Grant N/N203/402739. NH acknowledges support from the NASA Astrobiology Institute under Cooperative Agreement NNA09DA77A at the Institute for Astronomy (IfA), University of Hawaii, and NASA EXOB grant NNX09AN05G.

\section{References}

Agol, E., Steffen, J., Sari, R., \& Clarkson, W. 2005, MNRAS, 359, 567

Almeida, L. A., Jablonski, F., \& Rodrigues, C. V. 2013, ApJ, 766, 11

Beuermann, K. et al. 2010, A\&A, 521, 60

Beuermann, K., Dreizler, S., Hessman, F. V., \& Deller, J. 2012, A\&A A, 543, 138

Beuermann, K., Dreizler, S., \& Hessman, F. V. 2013, A\&A, 555, id. A133

Cincotta, P. M. \& Simó, C. 2000, A\&AS, 147, 205

Cincotta, P. M., Giordano, C. M., \& Simó, C. 2003, Physica D, 182, 151

Doyle, L. R. et al. 2011, Science, 333, 1602

Goździewski, K. et al. 2001, A\&̈A 378, 569

Goździewski, K. et al. 2012, MNRAS, 425, 930

Hinse, T. C., Lee, J. W., Goździewski, K., Haghighipour, N., Lee, C.-U., \& Scullion, E. M. 2012, MNRAS, 420, 3609

Horner, J., Marshall, J. P., Wittenmyer, R. A., \& Tinney, C. G. 2011, MNRAS, 416, 11

Horner, J., Wittenmyer, R. A., Hinse, T. C., \& Tinney, C. G. 2012, MNRAS, 425, 749

Horner, J., Hinse, T. C., Wittenmyer, R. A., Marshall, J. P., \& Tinney, C. G. 2012, MNRAS, 427,2812

Irwin, J. B. 1952, ApJ, 116, 218

Kozai, Y. 1962, AJ 67, 591

Lee, J. W. et al. 2009, AJ, 137, 3181

Migaszewski, C., Goździewski, K., \& Hinse, T. C. 2008, MNRAS, 395, 1204

Murray, C. D. \& Dermott, S. .F. 2000, Solar System Dynamics, Cambridge University Press

Orosz, J. A. et al. 2012a, ApJ, 758, 87

Orosz, J. A. et al. 2012b, Science, 337, 1511

Potter, S. B. et al. 2011, MNRAS, 416, 2202

Qian, S.-B. et al. 2011, MNRAS, 414, 16

Schwarz, R. et al. 2009, A\& A, 496, 833

Slonina, M., Goździewski, K., \& Migaszweski, C. 2012, in Arenou F., Hestroffer D., eds., Proc. Workshop "Orbital Couples: Pas de Deux in the Solar System and the Milky Way". Observatoire de Paris, Paris, p. 125

Welsh, W. et al. 2012, Nature, 481, 475

Wittenmyer, R. A., Horner, J., Marshall, J. P., Butters, O. W., \& Tinney, C. G. 2012 MNRAS, 419,3258

Wittenmyer, R. A., Horner, J., \& Marshall, J. P. 2013 MNRAS, 431, 2150 